\title{
Holographic Interferometry in Investigations of the Currenr Sheet Plasmas
}

\section{Anna G. Frank ${ }^{1}$}

Prokhorov Institute of General Physics of the Russian Academy of Sciences

Vavilov Str.38, Moscow, 119991, Russia

E-mail: annfrankefpl.gpi.ru

\section{Galya V. Ostrovskaya}

Ioffe Physical-Technical Institute of the Russian Academy of Sciences

Polytechnicheskaya Str. 26, St.-Petersburg,194021, Russia

E-mail: galya_ostremail.ru

Holographic interferometry is a highly efficient diagnostics for investigations of impulsive plasmas of large cross-sections. The double-exposure interferenceholographic method is based on the interference of two optical waves that passed through the object at different time moments, so that such interferogram records the changes of the object that appeared between two exposures.

Evolution of the current sheet plasmas has been studied by the holographic interferometry, and this research provides an excellent example of potentials of such diagnostics. As a result, a number of new phenomena have been revealed.

Recording the two-dimensional electron density distributions made it possible to visualize plasma sheets and to observe the effective plasma compression.

Observations of decreasing in the maximal plasma density and increasing in the thickness of the sheet made us conclude that the extra guide field exists inside the sheet.

Asymmetric and tilted plasma sheets revealed in the 3D magnetic configurations allowed us to suggest that the Hall currents are generated in the current sheet, and the sheet deformations result from interaction of the Hall currents with the guide field.

The fast non-stationary phenomena have been studied with the cine-holographic set-up, which could record five holograms in a single shot of the plasma device. This diagnostics allowed us to reconstruct the intriguing processes like explosive disruption of the current sheet.

First EPs Conference on Plasma Diagnostics - $1^{\text {st }}$ ECPD

14-17 April 2015,

Villa Mondragone, Frascati (Rome) Italy

\footnotetext{
${ }^{1}$ Speaker
} 


\section{Introduction}

Holography is a way of recording and reconstructing waves that is based on creating an interference pattern (hologram) formed by an object wave and a reference wave coherent with it. Subsequent interaction of the reference wave with the hologram structure gives rise to the reconstruction of the object wave.

The double exposure holographic interferometry consists in recording two holograms, which correspond to two different states of an object, onto the same photo-layer. The two waves that are holographic replicas of the waves, which existed at different moments, are reconstructed simultaneously and interfer. Holographic interferometry deals with the waves passing along the same path at different moments, and a holographic interferogram carries information about the changes of the object, which appeared between two exposures [1]. The holographic interferometry results in almost totally excluding distortions of the waves due to defects in the windows of plasma chamber and optical details.

Evolution and dynamics of the current sheet plasmas have been studied by the interference holographic methods, and this research provides an excellent example of potentials of such diagnostics.

A current sheet separates magnetic field lines of different (or opposite) directions, and magnetic reconnection processes in the current sheets are the basis for flare-type phenomena in the astrophysical and laboratory plasmas, such as solar and stellar flares, substormes in the magnetospheres of the Earth and planets, sawtooth instabilities in tokamaks, etc. [2,3].

\section{Experimental device CS-3D and the holographic set-up}

Experiments on formation and evolution of current sheets have been carried out with several devices of the "Current Sheet" series [4], the latest one being "CS-3D". The holographic set-up built around the device is shown in Fig.1 [5]. The basic components of the device CS-3D produce a quasi-steady 3D magnetic field with an X line, the initial plasma in this field, and the electric current along the $\boldsymbol{X}$ line, which gives rise to a current sheet. The structure of the magnetic field is as follows:

$$
\boldsymbol{B}=\left\{B_{x}{ }^{0} ; B_{y}{ }^{0} ; B_{z}{ }^{0}\right\}=\left\{h \cdot y ; h \cdot x ; B_{z}{ }^{0}\right\} .
$$

The transverse field gradient is $h=(0.5-2) \mathrm{kG} / \mathrm{cm}$, the uniform longitudinal (guide) field is $B_{z}{ }^{0}=(0-4.5) \mathrm{kG}$. The $\boldsymbol{X}$ line is aligned with an axis of the quartz vacuum chamber $18 \mathrm{~cm}$ in diameter, the space between two electrodes is $\Delta \mathrm{z}=60 \mathrm{~cm}$. The initial plasma density is $N_{e} \cong\left(2 \cdot 10^{14}-2 \cdot 10^{15}\right) \mathrm{cm}^{-3}$, the current amplitude is up to $J_{z}^{\max } \cong 55 \mathrm{kA}$, and its half-period is $\mathrm{T} / 2=6 \mu \mathrm{s}$. The magnetic field (1), the initial plasma, and finally the current sheet are practically uniform along the $z$ axis, so that we have significant benefits for application of the interferometer diagnostics, which yield the parameter values averaged over the line of sight, in our case along the $z$ axis. 
A holographic procedure is performed in two stages. In the first stage two holograms are successively recorded: one with the plasma, and another without it. In the reconstruction stage, this double-exposure hologram allows us to obtain the interference pattern of two waves that have passed the same way in successive instants.

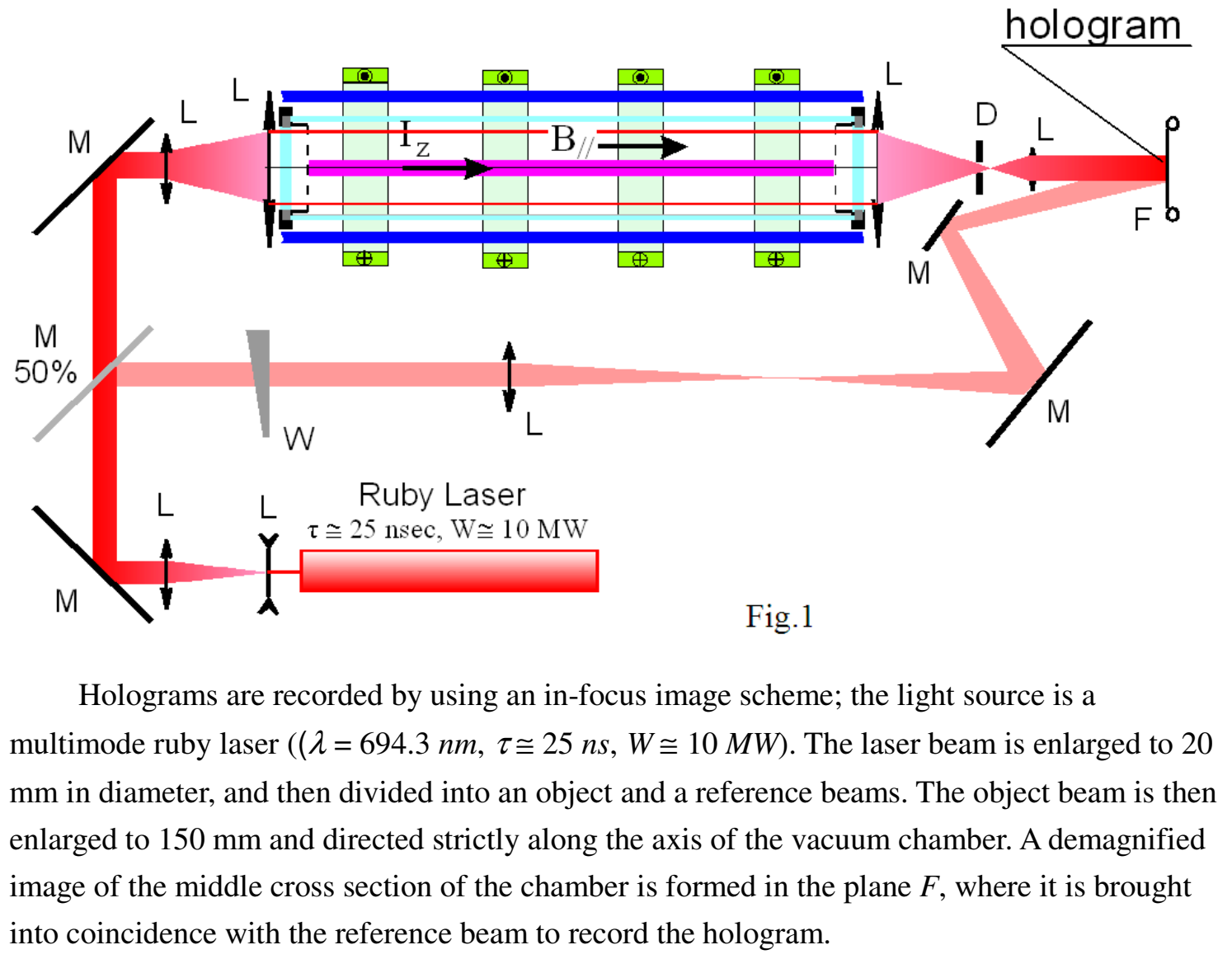

\section{Experimental results}

A typical interferogram and the $2 \mathrm{D}$ plasma density distribution $N_{e}(\mathrm{x}, \mathrm{y})$ obtained by processing the interferogram are shown in Fig.2. Because of the short duration of the laser radiation compared to the plasma processes, each interferogram represents a snapshot of the plasma at a fixed instant of time. It is evident, that the plasma sheet is a distinguished region, where the electron density $\mathrm{N}_{\mathrm{e}}$ is much higher than in the surrounding space. The sheet width, $2 \Delta x>6 \mathrm{~cm}$, is usually much larger than its thickness, $2 \Delta \mathrm{y}<1 \mathrm{~cm} . \mathrm{N}_{\mathrm{e}}$ decreases gradually along the sheet width, from the center to the edges, while the density decreases drastically in the $y$-direction normal to the sheet surface, where the density gradient is rather high.

Since $\cong$, the shifts of an interference fringe are proportional to $\mathrm{N}_{\mathrm{e}}(\mathrm{x}, \mathrm{y})$,

$$
\mathrm{k}(\mathrm{x}, \mathrm{y})=\Delta \varphi / 2 \pi=4.49 \cdot 10^{-14} \cdot \lambda \cdot \mathrm{N}_{\mathrm{e}}(\mathrm{x}, \mathrm{y}) \cdot \Delta \mathrm{z},
$$

and a shift by one fringe $(\mathrm{k}=1)$ corresponds to $\mathrm{N}_{\mathrm{e}}=5.33 \cdot 10^{15} \mathrm{~cm}^{-3}$ for the CS-3D device. The maximal electron density, $N_{e}^{\max }=(1-1.5) \cdot 10^{16} \mathrm{~cm}^{-3}$, is located in the current sheet mid-plane, and this value is 5-15 times the initial density [6]. 

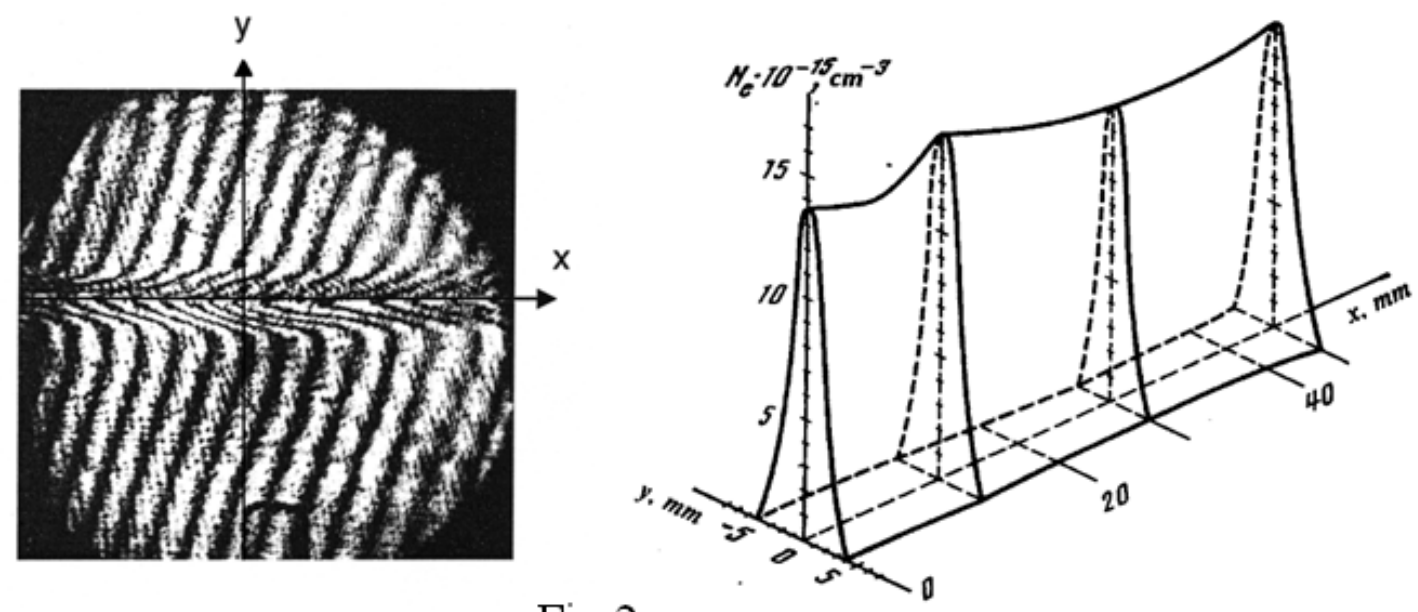

Fig. 2

The time dependence of the maximal plasma density in the central region of the sheet makes it evident that the plasma is effectively compresed in the course of the sheet formation. At the same time, as $B_{z}{ }^{0}$ increases, $N_{e}{ }^{\max }$ decreases, and the sheet thickness increases, while the total amount of electrons per $1 \mathrm{~cm}$ of the sheet width (integrated along the sheet thickness) depends only slightly on $B_{z}{ }^{0}$. The stronger the guide field, the less efficient the plasma compression, in other words, there is a tendency toward the behavior of uncompressible plasma. We have shown that it is caused by the enhancement of the guide field inside the sheet above its initial value $B_{z}^{0}[6,7]$.
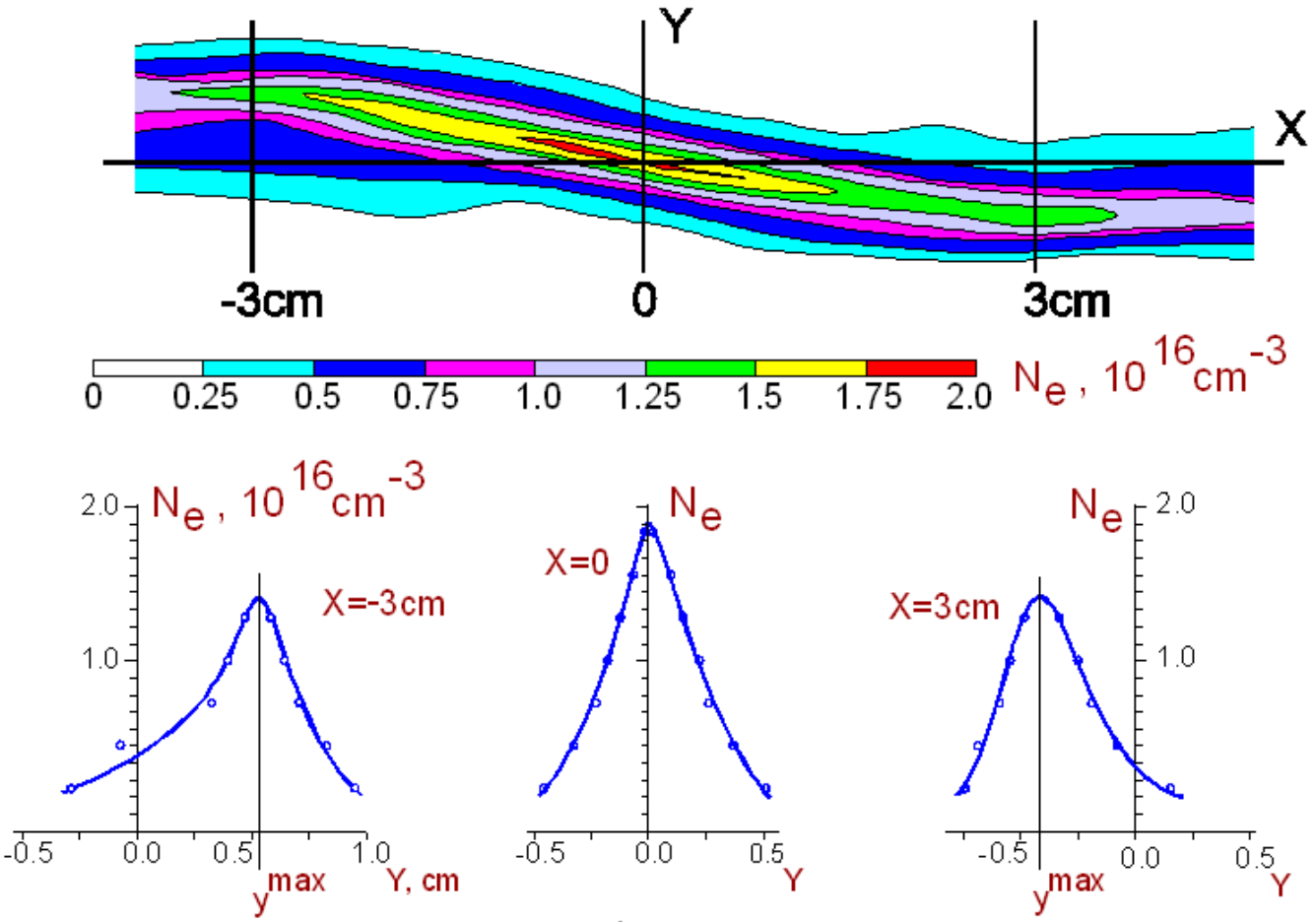

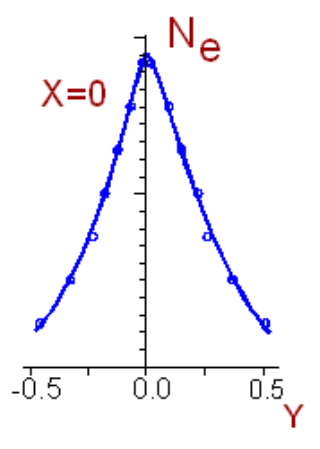

Fig. 3

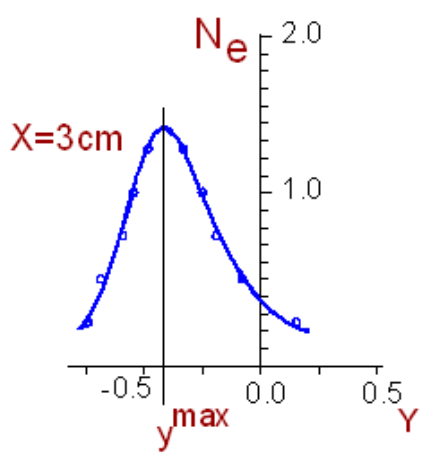


In 3D magnetic configurations we have observed an impressive effect: formation of tilted and asymmetric plasma sheets, which appear only in the presence of the guide field $B_{z}{ }^{0}$, Fig. 3 [8]. The angle of the sheet tilting changes the sign when we reverse the direction of the guide field. In addition, the tilting is more pronounced if the sheet is formed in plasmas with heavier ions. These results made us conclude that the sheet deformations appear due to generation of the Hall currents and interaction of the Hall currents with the guide field $B_{z}{ }^{0}$ [8].

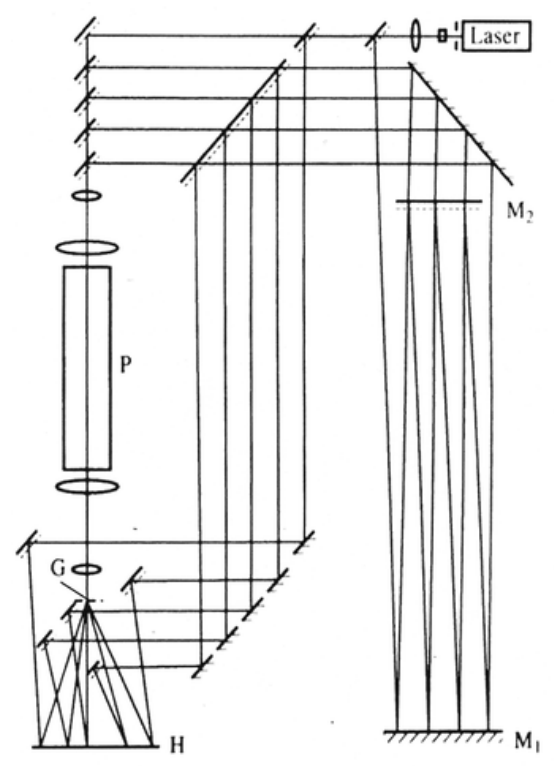

Fig.4

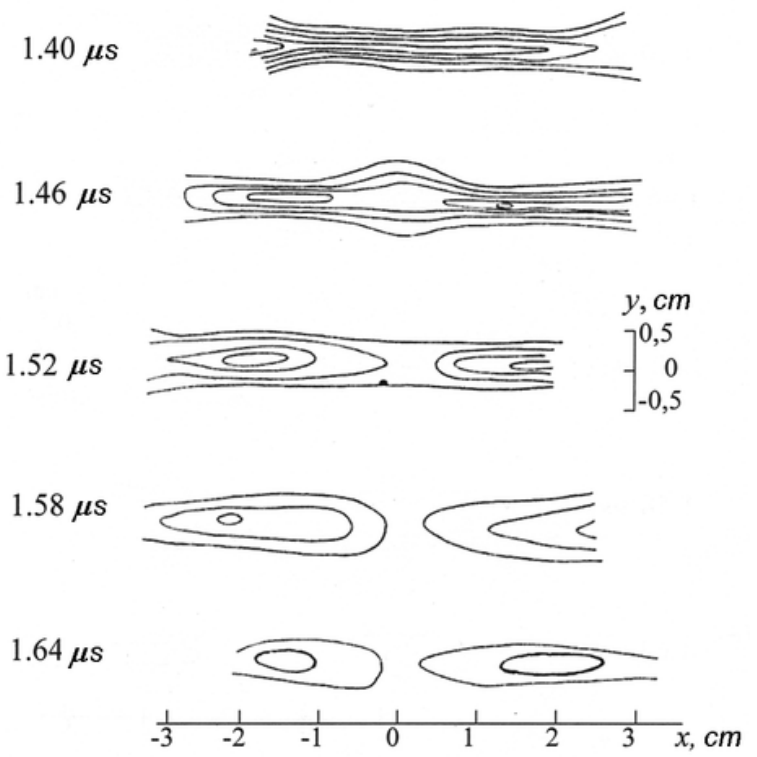

Fig.5

The fast non-stationary phenomena in the current sheets have been studied with specially designed cine-holographic set-up, which included an optical delay line to produce a series of five light pulses shifted in time on $60 \mathrm{~ns}$, Fig.4 [9]. Each of the light beams is divided into an object beam and a reference beam, and all five object beams are directed through the plasma strictly along the z-axis. In the case of five superimposed holograms, their quality would be rather poor. To improve the situation, the object beams, which coincide in space, are splitted into several parts by a diffraction grating and are directed to different regions of a phtosensetive material. Each region is illuminated by only one reference beam, forming a single hologram, while the rest object beams produce incoherent background. This method allowed $\approx 10$-fold increase in the diffraction efficiency of a hologram as compared with the superimposed holograms [1].

The cine-holographic set-up makes it possible to obtain five double-exposure holographic interferograms in a single shot of the plasma device, with the delay time $60 \mathrm{~ns}$ between successive interferograms. This diagnostics provides observations of fast impulsive processes of a great interest like explosive disruption of a current sheet, Fig.5 [10]. 


\section{Summary}

The methods of the holographic interferometry made a significant contribution in the research on the dynamics and evolution of the current sheet plasmas and magnetic reconnection processes. As a result, a number of new phenomena has been revealed.

Recording the $2 \mathrm{D}$ electron density distributions makes it possible to visualize the plasma sheets and to observe the effective (five- to tenfold) plasma compression.

A decrease in the maximal plasma density simultaneously with an increase in the thickness of the sheet produced in a 3D magnetic configuration, in the presence of the guide field, allowed us to conclude that the extra guide field exists inside the sheet.

Revealing of asymmetric and tilted plasma sheets formed in the 3D magnetic fields, in plasmas with heavy ions, gave rise to an idea about generation of the Hall currents in a current sheet, and the sheet deformations due to interaction of the Hall currents with the guide field.

The fast non-stationary phenomena have been studied with specially designed cineholographic set-up, which allowed us to obtain five holographic interferograms in a single shot of the plasma device. As a result, it became possible to reconstruct in details not only the formation process, but also the process of explosive disruption of the current sheet.

The work is supported in part by the Russian Foundation for Basic Research (project no 15-02-03644).

\section{References}

[1] G.V. Ostrovskaya, Yu.I. Ostrovsky, Progress in Optics, 22, 199 (1986)

[2] S.I. Syrovatskii, Annu. Rev. Astron. Astrophys. 19, 163 (1981)

[3] E.R. Priest, T. Forbes, Magnetic reconnection. MHD theory and applications, Cambridge University Press, Cambridge, UK, 2000

[4] A.G. Frank, Physics-Uspekhi 180(9), 941 (2010)

[5] S.Yu. Bogdanov, V. S. Markov, A.G. Frank, et al., Plasma Phys. Rep. 28, 549 (2002)

[6] A.G. Frank, S.Yu. Bogdanov, V.S. Markov, et al., Phys. Plasmas 12, 052316 (2005)

[7] A.G. Frank, S.G. Bugrov, V.S. Markov, Phys. Lett. A 373, 1460 (2009)

[8] A.G. Frank, S.Yu. Bogdanov, G.V. Dreiden, et al., Phys. Lett. A 348, 318 (2006)

[9] G.V. Dreiden, V.S. Markov, G.V. Ostrovskaya, et al., Sov. J. Plasma Phys. 4, 14 (1978)

[10] S.Yu. Bogdanov, G.V. Dreiden, A.G. Frank, et al., Physica Scripta 30, 282 (1984) 
Title (or short title)

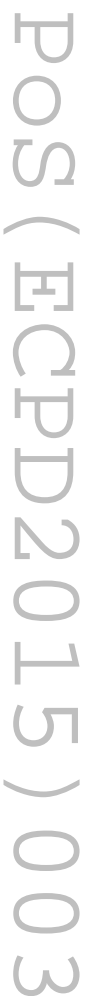

